\title{
Prostaglandin E2 Levels in the Evaluation of Management of Osteoarthritis Using Pulsed Electromagnetic Field: An Interventional Study
}

\author{
Sajuni I. ${ }^{1}$, Karthika M. ${ }^{1}$, Sujatha B. ${ }^{2}$, Anbarasi R. ${ }^{1}$, S. Vishnuprasaath ${ }^{3}$, Vijayalakshmi B. ${ }^{4}$ \\ ${ }^{1}$ Assistant Professor, ${ }^{2}$ Professor and Head, Department of Physiology, Government Pudukkottai Medical College \\ \& Hospital, Pudukkottai, ${ }^{3}$ Tutor, ESIC Medical College, K.K. Nagar, Chennai, ${ }^{4}$ Professor \& Head, Department of \\ Physiology, Saveetha Medical College \& Hospital, Thandalam, Chennai
}

\begin{abstract}
Knee pain is one of the most frequent musculoskeletal problems among the middle-aged and elderly people and 'Osteoarthritis' is the widespread clinical pathology of the knee.

Objective: The present study was conducted to evaluate whether low frequency Pulsed Electro Magnetic Field (PEMF) therapy is effective in patients with osteoarthritis knee using surface Electromyography (sEMG) of vastusmedialis.

Method: The participants were subjected to Pulsed Electromagnetic Field Therapy using the PULSATRON instrument designed by Madras Institute of Magnetobiology, Anna nagar. They were given PEMF therapy of $10 \mathrm{~Hz}$ for 60 minutes/day for 21 days with a break after every 6 days. Serum PGE2 levels are measured by invitro ELISA method. Serum Prostaglandin E2 levels are estimated before and after PEMF therapy.

Results: The study shows that Low frequency PEMF $(10 \mathrm{~Hz})$ can be used as a treatment modality for Osteoarthritis. PEMF therapy, when given for a sufficient time (21 days) has shown to reduce the inflammation by decreasing the levels of the inflammatory marker Prostaglandin E2.Serum PGE2 levels were found to be significantly decreased $(\mathrm{p}<0.001)$ in the study group following the Pulsed Electro Magnetic Field (PEMF) therapy.
\end{abstract}

Keywords: Low frequency PEMF, Serum PGE2.

\section{Introduction}

Osteoarthritis causes degradation of joints, articular cartilage and subchondral bone ${ }^{1}$.About $40 \%$ ofthe elderly Indian Population suffer from osteoarthritis, $2 \%$ among them experience crucial pain and disability. ${ }^{2}$ Females are affected more severely with osteoarthritis with more number of joints involved particularly the knee

\section{Corresponding Author:}

\section{Dr. Karthika M.}

Assistant Professor, Department of Physiology, Dhanalakshmi Srinivasan Medical College \& Hospital, Perambalur and hand. ${ }^{3}$ Osteoarthritis is broadly classified into two main categories - Primary osteoarthritis and Secondary osteoarthritis. $^{4}$ The Primary form of osteoarthritis occurs due to an idiopathic etiology. The secondary forms of osteoarthritis occur as a consequence of some underlying pathology. The common etiologies include: Congenital, Post surgery, injury, Endocrine, Metabolic, Rheumatologic and Neurological.

Patients of osteoarthritis knee present with knee pain, stiffness, swelling of the joint, cracking with joint movement and decreased function of the joint. A longstanding osteoarthritis knee left untreated leads to severe functional disability. The limitations are due to the cartilage defect, erosion and fusion of the joint and muscle stiffness. Most of the treatments at present are 
aimed at pain reduction, improving the joint stiffness and maintenance of joint function.

Inflammation is the defense mechanism of the body in response to the injury involved in Osteoarthritis. It is an advantageous event which results in elimination of pathogenic factors and restoration of normal function of the tissues. The failure of the acute phase of inflammation to terminate the deterioration will progress to chronic inflammation andtissue damage. ${ }^{5}$ The inflammatory mediator $\mathrm{PGE}_{2}$ isresponsible for all three established signs of inflammation - redness, swelling and pain. Redness and edema are due to the fact of increased blood supply to the inflamed tissue produced by the local arteriolar dilatation. Pain results from theaction of $\mathrm{PGE}_{2}$ on the sensory neurons at the peripheral and central sites. ${ }^{6}$

Pulsed Electromagnetic Field (PEMF) is a modern therapeutic device used in treating many clinical conditions. Though the clinical application of PEMF is still under controversy, it is being widely used as a treatment modality over the past two decades and has shown to decrease pain, inflammation and stiffness in patients with osteoarthritis. ${ }^{7}$ The mechanism behind this is the increase in blood supply to peri-articular compartment due to synthesis of nitric oxide. ${ }^{8}$

This study focuses on evaluating whether the low frequency and low-intensity Pulsed Electromagnetic Field (PEMF) therapy is effective in osteoarthritis by assessment of inflammation by estimating the level of Prostaglandin PGE2 before and after administration of Pulsed Electro Magnetic Field Therapy.

\section{Materials and Method}

An interventional study was conducted in the Institute of Physiology and Experimental Medicine, Madras Medical College in collaboration with the Institute of Geriatrics, Rajiv Gandhi Government General Hospital, Chennai from April 2015 to March 2016. Fifty patients between 50 to 70 years of age with osteoarthritis knee having symptoms for atleast one year duration were included in the study after obtaining Ethical approval from Institutional Ethics Committee (IEC), Madras Medical College, Chennai. Patients with chronic disorders were excluded from the study.
Methodology: The participants were subjected to Pulsed Electromagnetic Field Therapy using the PULSATRON instrument designed by Madras Institute of Magnetobiology, Anna nagar. They were given PEMF therapy of $10 \mathrm{~Hz}$ for 60 minutes/day for 21 days with a break after every 6 days (protocol designed by Madras Institute of Magnetobiology). Blood samples are collected under strict aseptic precautions by venepuncture of antecubital vein and serum is separated by centrifugation. Serum PGE2 levels are measured by invitro ELISA method. Serum Prostaglandin E2 levels were estimated before and after PEMF therapy.

Statistical Analysis: After collection, data were checked for consistency and completeness. Then the data was entered in database Statistical Package for the Social Sciences (SPSS) software version 21. The Paired Student's $t$ test was carried out to compare the mean of variables before and after administration of Pulsed Electro Magnetic Field therapy.

\section{Results}

The present study was done to evaluate whether low frequency Pulsed Electro Magnetic Field (PEMF) therapy is effective in patients with osteoarthritis knee by estimating the levelof Prostaglandin $\mathrm{PGE}_{2}$ before and after administration of Pulsed Electro Magnetic Field Therapy.

\section{Table 1: Baseline Parameters}

\begin{tabular}{|c|l|c|}
\hline S.No. & Variable & Mean \pm SD \\
\hline 1. & Age (in years) & $62.08 \pm 4.36$ \\
\hline 2. & Duration of osteoarthritis (in years) & $3.46 \pm 1.53$ \\
\hline 3. & BMI & $27.57 \pm 2.59$ \\
\hline
\end{tabular}

The mean age of the individuals included in the present study was $62.08 \pm 4.36$ years with the 50 to 70 years. The mean duration of symptoms of osteoarthritis in study subjects was $3.46 \pm 1.53$ years. The average BMI was found to be $27.57 \pm 2.59$.

Blood samples are collected under strict aseptic precautions by means of venepuncture of antecubital vein and serum is separated by centrifugation. Serum PGE2 levels are measured by invitro ELISA method. Serum Prostaglandin E2 levels were estimated before and after PEMF therapy. 
Table 2: Comparison of mean values of the of PGE2 levels before and after PEMF therapy

\begin{tabular}{|l|l|l|c|c|c|}
\hline Variable & Group & N & Mean & SD & P -Value \\
\hline \multirow{2}{*}{ Prostaglandin E2 levels } & Before PEMF & 50 & 471.16 & 104.94 & $<0.001 * * *$ \\
\cline { 2 - 6 } & After PEMF & 50 & 290.65 & 74.14 \\
\hline
\end{tabular}

*** $\mathrm{P}-$ Value $<0.001$ Very Highly Significant

Table 2 shows that the Serum PGE2 levels were found to be significantly decreased $(p<0.001)$ in the study group following the Pulsed Electro Magnetic Field (PEMF) therapy.

\section{Discussion}

The present study revealed that Low frequency PEMF $(10 \mathrm{~Hz})$ can be used as a treatment modality for Osteoarthritis, when given for a sufficient time (21 days).Similar results are shown by various studies found in the literature. Finietal stated that PEMF being applied at $75 \mathrm{~Hz}, 1.6 \mathrm{mT}, 6 \mathrm{hrs}$ perday for three months proved to prevent the development of osteoarthritis in aged guinea pigs. ${ }^{9}$ Aaronand Ciomboretal examined the effects of PEMF in a decalcified bone matrix. The observations were noted with the increase in matrix synthesis which is stimulated by the proliferation of mesenchymal cells. ${ }^{10}$ Ciombor et al had proved the enhanced synthesis of cartilage under the effect of themagnetic field applied at a burst of $4.5 \mathrm{~ms}$ duration and further repeated at 15 bursts. ${ }^{11}$ De Mattei et al observed the anabolic effects of PEMF in the cartilage at different ranges of exposure length (1,4,9 and $24 \mathrm{~h})$, different frequencies (2, 37,75 and $110 \mathrm{~Hz})$ and magnitudes $(0.5,1,1.5,2 \mathrm{mT}) .^{12}$

Although the osteoarthritis is a non- inflammatory disease, various cytokines, prostaglandins and reactive oxygen species are believed to play important roles in pathogenesis. ${ }^{13}$ The degenerative actions of PGE2 on the chondrocytes are exerted through the EP4 receptor which activates the enzyme adenylcyclase through $G$ proteins resulting in the accumulation of intracellular cyclic adenosine monophosphate. ${ }^{14}$ Lawand et al stated that Pain in osteoarthritis is contributed mainly by factors like increase in inflammation occurring at early stages of arthritis and destruction of articular cartilage. ${ }^{15}$ The genetic studies conducted by Sato et al proved that there was an elevation in the expression of the terminal synthase microsomal prostaglandin synthase (mPGEs) which was essentialfor the COX-2 derived PGE2 production in the diseased cartilage. ${ }^{16}$ PGE2 induced pain reduction was seen during magnetic field exposure by modulating the exogenous and endogenous opioid systems in a study conducted by M.Kavaliers et al. ${ }^{17}$ Senthil Kumar V et al observed a decrease in lysosomalactivities in arthritis rats on exposure to PEMF. ${ }^{18}$ Studies done by Chang et al noticed a reduction in levels of TNF- $\alpha$ and IL- 6 in ovariectomised rats which were exposed to PEMF for 7 days with different intensities of electric field. ${ }^{19}$

\section{Conclusion}

The study shows that Low frequency PEMF $(10 \mathrm{~Hz})$ can be used as a treatment modality for Osteoarthritis. PEMF therapy, when given for a sufficient time (21 days) has shown to reduce the inflammation by decreasing the levels of the inflammatory marker, Prostaglandin E2. Hence, PEMF, a novel approach, can be used in treating chronic osteoarthritis knee adjunctive to the pharmacotherapies that are currently in use.

Limitation of the study: Further studies including a large number of diabetic and hypertensive participants and assessment of the efficacy of PEMF therapy by other markers of the disease should be done.

\section{Source of Funding: Self}

\section{Conflict of Interest: Nil}

Acknowledgment: We acknowledge great help received from the scholars whose articles are cited and included in the references of this manuscript.

\section{References}

1. Di Cesare P, Abramson S, Samuels J. Pathogenesis of osteoarthritis.In: Firestein GS, Kelley WN, eds. Kelley's Textbook of Rheumatology .8th ed. Philadelphia, Pa.: Saunders Elsevier; 2009: 152540.

2. Sharma MK, Swami HM, Bhatia V, Verma A, Bhatia SP, Kaur G. 2007. An Epidemiological Study of Correlates of Osteo-Arthritis in Geriatric 
Population of UT Chandigarh. Indian J Community Med;32:77-8.

3. Parazzini F; Progretto Menopausa Italia Study Group. Menopausal status, hormone replacement therapy use and risk of self-reported physiciandiagnosed osteoarthritis in women attending menopause clinics in Italy. Maturitas 2003 20;46:207-12.

4. Moskowitz RW. Osteoarthritis symptoms and signs. Osteoarthritis Diagnosis and Management; 5th edtition,WB Saunders 1993. pp. 255-261.

5. Nathan C. Points of control in inflammation. Nature. 2002;420:846-885.

6. Narumiya S, FitzGerald GA. Genetic and pharmacological analysis of prostanoid receptor function. J Clin Invest. 2001;108:25-30.

7. Jari PA, TaruV,Markkuk, ölavi A: Activation atlumbarparsapinal and abdominal muscles during therapeutic exercises in chronic low back pain patients. Arch of Phy. Med. and Rehab 2004; 85: $823-823$.

8. Fischer G, Pelka RB, Barovic J: Adjuvant treatment of knee osteoarthritis with weak pulsing magnetic fields. Results of a placebo-controlled trial prospective clinical trial. Z OrthopIhreGrenzgeb 2005;143:544-550.

9. Fini M, Giavaresi G, Torricelli P, Cavani F, Setti S, Cane V \& Giardino R, Pulsed electromagnetic fields reduce knee osteoarthritic lesion progression in the aged Dunkin Hartley Guines pig, J Orthop Res, 23 (2005) 899.

10. Aaron RK \& CiomborDMcK, Acceleration of experimental endochondral ossification by biophysical stimulation of the progenitor cell pool, J Orthop Res, 14 (1996) 582.

11. Ciombor DMcK, Aaron RK, wang S and Simon B, Modification of osteoarthritis by pulsed electromagnetic field-morphological study. Osteoarthritis and Cartilage, $11:(2003) 455$.
12. De Mattei M, Caruso A, Pezzetti F, Pellati A, Stabellini G, Sollazzo V \& Traina GC, Effects of pulsed electromagnetic fields on human chondrocyte proliferation, Connect Tissue Res, 42 (2001) 269.

13. Abramson, S. B., M. Attur, and Y. Yazici. 2006. Prospects for disease modification in osteoarthritis. Nat. Clin. Pract. Rheumatol. 2: 304-312.

14. Pierce KL, Fujino H, Srinivasan D, Regan JW. Activation of FP prostanoid receptor isoforms leads to Rho-mediated changes in cell morphology and in the cell cytoskeleton. J Biol Chem. 1999;274:35944 35949

15. Lawand NB, McNearney T, Westlund KN. Amino acid release into the kneejoint: keyrole innociception and inflammation.Pain. 2000;86(1):69e74.

16. Sato, T., K. Konomi, R. Fujii, N. Yagashita, M. Yokouchi, S. Komoya, M. Beppu, K. Nishioka, and T. Nakajima. 2007. IL-1 induced MMP-13 expression is regulated by prostaglandin E2-EP2 signalling in human osteoarthritic chondrocytes. Arthritis Rheum. 54: S317.

17. M.Kavaliers and KP Ossenkopp, "Opioid systems and Magnetic Field Effects in the Land snail, Cepaeanemoralis". Biological Bulletin, 180, 1991, pp.301-309.

18. Senthil Kumar V, Ashok Kumar D, Kalaivani K, Gangadharan AC, Narayana raju KVS, Thejomoorthy P, Murali Manohar B \& Puvanakrishnan R, Optimization of pulsed electromagnetic field therapy for management of arthritis in rats. Bioelectromag, 26 (2005)431.

19. Chang K, Chang WH, Wu ML \& Shin C, Effects of different intensities of extremely low frequency pulsed electromagnetic fields on formation of osteoclast-like cells, Bioelectromag, 24 (2003) 431. 